NOTE MÉTHODOLOGIQUE

Centre de Recherche en Psychologie de la Connaissance, du Langage et de l'Émotion

Université de Provence'

\title{
COMMENT ÉTUDIER LE COÛT ET LE DÉROULEMENT DE LA RÉDACTION DE TEXTES LA MÉTHODE DE LA TRIPLE TÂCHE UN BILAN MÉTHODOLOGIQUE
}

par Annie PIOLAT et Thierry OLIVE

$S$ UMMARY : How can the process and cost of writing texts be studied? The triple task methodology

This methodological note has three goals. The first is to describe the various uses of the triple task paradigm (writing; quick reactions, think aloud protocols) proposed by Kellogg (1986, 1987b) and Levy and Ransdell (1994, 1995). The dependent variables related to this paradigm are examined. The second aim is to evaluate the validity of the paradigm by measuring the task's reactivity and that of verbalisation. The third goal is to offer a short review of findings related to the use of the paradigm. We conclude by showing the efficacy of the triple-task paradigm for the investigation of text writing.

Key words : writing, processes, cognitive effort, time processing, triple task.

\section{INTRODUCTION}

Depuis plus de vingt ans, de nombreux chercheurs ont souligné la rareté des recherches sur la rédaction de textes, laquelle est attribuée à la difficulté d'étudier l'activité de production

1. 29, avenue Robert-Schuman, 13621 Aix-en-Provence Cedex 1. E-mail annie.piolat@up.univ-mrs.fr; thierry.dive@univ-poitiers.fr 
comparativement à celle de compréhension (cf., par ex., Bonin et Fayol, I996). Cette difficulté aurait plusieurs origines (contrôle des sources de variations, éventail très large des solutions produites par les rédacteurs, etc.). Ainsi, l'étude de la rédaction de textes échapperait encore aux exigences de l'investigation expérimentale par un manque de méthodes propices à fournir des faits reproductibles.

Cette conception transparaît toujours dans des synthèses récentes, mais le point de vue est sensiblement différent. Toutefois, ce n'est plus entre l'étude de la compréhension et l'étude de la production qu'une hiérarchie est établie selon des critères quantitatif et qualitatif, mais plutôt entre celle de la production verbale orale et celle de la production verbale écrite qu'elle est maintenant établie. À côté de l'ampleur des modélisations et des travaux réalisés en production verbale orale, l'étude de la production verbale écrite paraîtrait bien indigente. Pour s'en convaincre, il suffit de lire des ouvrages de synthèse dans le domaine de la production verbale (Bock, 1996 ; Gernsbacher, 1994 ; Levelt, 1989). Les modèles et les méthodes concernant la production écrite sont, d'après Fayol (I997), loin d'avoir la puissance de ceux développés dans le champ de la production verbale orale.

Toutefois, comme en témoignent des ouvrages récents proposant une approche cognitive de la rédaction de textes (Levy et Ransdell, 1996 ; Rijlaarsdam, van den Bergh et Couzijn, 1996), des expérimentations de qualité sont maintenant disponibles même si, comme le souligne Levy (1997), le nombre de recherches pourrait être largement amplifié. Aussi, l'objectif de cette revue est de montrer que le déroulement de l'activité rédactionnelle peut, malgré sa complexité, être étudié de façon pertinente, les paradigmes de recherche étant disponibles et éprouvés (pour un bilan plus exhaustif des méthodes utilisées pour étudier la rédaction de textes, cf. Coirier, Gaonac'h et Passerault, 1996 ; Fayol, 1997 ; Piolat et Pélissier, 1998).

Depuis les propositions de Hayes et Flower (1980), trois sous-processus basiques de la rédaction de textes sont l'objet de recherches : la planification, la mise en texte et la révision. A part quelques exceptions (Kellogg, 1987b, I988, 1994 ; Levy et Ransdell, 1994, 1995 ; Olive, 1997 ; Penningroth et Rosenberg, 1995 ; Piolat, Roussey, Olive et Farioli, 1996 ; Ransdell et Levy, 1996), rares sont les chercheurs qui ont tenté de déterminer coin- 
ment, au cours de la rédaction, le temps et l'effort cognitif étaient dévolus à chacun de ces processus.

Le programme de recherches proposé par Flower et Hayes (1980) depuis vingt ans concerne la mise en évidence de l'enchaînement récursif des processus rédactionnels et l'éva luation de la charge que leur activation impose au système de traitement. Afin de guider le développement de modèles pouvant rendre compte de la qualité rédactionnelle, il est utile de disposer de données empiriques plus pertinentes sur la façon dont les individus allouent leurs ressources attentionnelles aux processus qu'ils mobilisent tout au long de la rédaction.

Le paradigme de la triple tâche a été mis au point pour fournir des observables aptes à concrétiser ces objectifs de recherches. Dans cet article, trois aspects seront abordés. Dans un pre mier temps la méthode mise au point par Kellogg (1987a et $b$ ) et la variante qu'en proposent Levy et Ransdell (1994, 1995) seront présentées. Dans un deuxième temps, la comparaison de ces deux paradigmes sera faite en examinant les recherches qui statuent sur leur validité respective. Enfin, un rapide bilan des faits expérimentaux recueillis à l'aide de ces deux variantes du paradigme de la triple tâche sera proposé.

\section{DESCRIPTION DU PARADIGME DE LA TRIPLE TÂCHE}

\section{1. LE PARADIGME DE KELLOGG}

Afin d'évaluer l'effort cognitif associé aux processus impliqués dans la rédaction de texte ainsi que la façon dont le rédacteur mobilise, pas à pas, ces processus, Kellogg $(1987 b, 1988)$ a employé une procédure expérimentale imposant au rédacteur de réaliser trois tâches. En plus de sa tâche principale (rédiger un texte), le rédacteur doit effectuer, dans le même temps, deux tâches ajoutées : réagir vite à des stimuli sonores et opérer, après chaque réaction rapide, une rétrospection dirigée.

Ainsi, pendant qu'il compose, le participant perçoit des stimulus sonores auxquels il doit, à chaque fois, rapidement réagir. Avant de poursuivre son activité rédactionnelle, le rédacteur doit, après chaque réaction rapide, qualifier ce qu'il était en 
train de faire (Planifier. Mettre en texte, Réviser ou Autre). Cette catégorisation dirigée permet d'associer à chacun des temps de réaction le processus qui a été interrompu. Le temps de réaction traduit l'importance de l'effort cognitif associé au processus rédactionnel dont le déroulement a été interrompu par le signal sonore. Autrement dit, par rapport à une mesure de référence où la tâche ajoutée est réalisée séparément (mesure du temps de réaction moteur moyen), la variation des temps de réaction donne une indication sur la variation des ressources utiles à la tâche principale. Les données issues de la catégorisation fournissent des informations sur la mobilisation pas à pas des différents processus rédactionnels.

\subsubsection{Procédure}

Plus précisément, la procédure expérimentale comprend trois phases : 1 / l'entraînement à la rétrospection dirigée ; 2 / l'entraînement à la stimulation sonore et le recueil du temps de réaction moteur de base; 3 /la réalisation de la tâche principale d'écriture associée aux deux autres tâches (réaction et rétrospection). Lorsque la phase 3 dure environ une demiheure, la durée totale de l'expérience est d'environ une heure trente.

Durant la première phase de l'expérience, le participant est entraîné à l'activité de rétrospection dirigée. L'expérimentateur commence par lui donner la définition des processus rédaction nels (Planifier, Mettre en texte, Réviser) en les exemplifiant avec des extraits de protocoles verbaux (pensées émises à haute voix) produits par des rédacteurs. Ensuite, le participant catégorise lui-même plusieurs exemples de pensées censées avoir accompagné l'activité d'un rédacteur (e.g. «Je me demande avec quelle sorte d'argument je vais terminer mon texte »; «J'utilise ce terme auquel je tiens »). En cas d'erreur de classification, une discussion avec l'expérimentateur permet de faire une mise au point. Enfin, le rédacteur est informé qu'avec la catégorie « Autre », il pourra désigner toutes les autres pensées qui ne sont pas en relation avec son activité rédactionnelle (ex. « Penser à passer à la bibliothèque après avoir fini l'expérience »).

Dans la deuxième phase de l'expérience, le participant est informé qu'il entendra, pendant son activité de composition, 
une série de signaux sonores et qu'il devra répondre, à chaque fois, le plus rapidement possible en disant «stop ». Le participant est ensuite soumis à une série de 30 stimulations sonores. Les sondes sonores sont délivrées selon une cadence de distribution aléatoire dont l'intervalle varie de cinq à quinze secondes. Les cinq premiers temps de réaction, considérés comme des essais, ne sont pas pris en compte dans le calcul du temps de réaction moteur de base.

Durant la troisième phase de l'expérience, le thème rédactionnel est lu au participant. La consigne concernant le mode de composition du texte est aussi donnée ; par exemple, selon les objectifs de la recherche, la consigne peut préciser que l'aspect fini du texte n'est pas important tant qu'il reste lisible car pour exposer au mieux ses idées, le participant peut à tout moment modifier son texte. Souvent, le temps d'écriture n'est pas limité. L'expérimentateur demande au rédacteur de rester concentré sur le texte qu'il va rédiger malgré les deux tâches ajoutées. Pendant qu'il rédige, le participant entend toutes les trente secondes environ (la cadence de stimulation varie de façon aléatoire de quinze à quarante-cinq secondes. $\boldsymbol{N B}$. - Kellogg a aussi utilisé des cadences plus lentes) des sondes sonores auxquelles il doit répondre le plus rapidement possible. Après chaque interruption de la rédaction provoquée par un signal sonore, le rédacteur désigne son activité rédactionnelle en cours (Planifier, Mettre en Texte, Réviser).

\subsubsection{Variables dépendantes}

Différentes variables dépendantes sont utilisées par Kellogg. Pour analyser le coût cognitif des processus rédactionnels ainsi que leur mobilisation, deux variables ont été considérées : le temps de réaction pondéré et la fréquence de désignation des processus

pour les trois processus rédactionnels un temps de réaction pondéré moyen (T.Rp en millisecondes) par participant est calculé. Ce temps de réaction pondéré (appelé aussi par Kel$\log$ 《 «emps de réaction interférent ») est établi à partir de la moyenne des différences entre chacun des temps de réaction mesurés pendant l'activité d'écriture et le temps de réaction moteur de base du participant calculé préalablement ; 
pour chacun des participants, une fréquence moyenne de désignation des processus est calculée. De plus, pour chacun des participants, une partition en trois durées équivalentes de son temps total de production est opérée. Cette partition permet, d'une part, d'analyser le pattern des processus rédactionnels dans chacun de ces tiers, et, d'autre part, d'analyser l'évolution de la mobilisation des processus d'un tiers à l'autre.

Enfin, différents indices permettent d'évaluer l'efficience et la qualité des textes produits par chacun des rédacteurs (nombre de mots du texte achevé, débit, complexité syntaxique, nombre de révisions, etc., cf. Kellogg, 1987a).

\subsection{LE PARADIGME DE LEVY ET RANSDELL}

Le paradigme de la triple tâche mis au point par Levy et Ransdell (1994, 1995) est inspiré de celui de Kellogg (1987b). Toutefois, le contexte informatique dans lequel les données sont recueillies est particulièrement sophistiqué.

Afin de caractériser le temps et l'effort impartis aux processus rédactionnels, Levy et Ransdell confrontent pour chacun des rédacteurs ses protocoles écrits (le ou les textes(s) qu'il a produit(s) à l'aide d'un traitement de texte, cf. Ransdell, 1990), ses protocoles verbaux (enregistrement des verbalisations émises pendant la rédaction, cf. Ransdell, 1995) et ses protocoles d'allocation de ressources (enregistrement des réactions rapides à des stimulations sonores pendant la rédaction). Selon ces auteurs, cette confrontation facilite la définition opérationnelle de quatre sous-processus basiques : la planification, la mise en texte, le réexamen, la révision. En effet, les protocoles écrits fournissent l'enregistrement de tout le texte tel qu'il a été élaboré et corrigé, apportant ainsi plus d'informations que le simple produit écrit final. Les protocoles verbaux synchronisés dans le temps avec le texte en cours d'écriture contiennent des commentaires qui permettent de déterminer si les pauses produites pendant la rédaction sont associées au réexamen du texte déjà écrit ou à la planification du futur texte (cf. les propositions de Flower et Hayes, 1981 ; Kaufer, Hayes et Flower, 1986). De cette manière, les processus de réexamen et de révision sont facilement différenciés à l'aide du contenu des pensées à haute voix et 
de la présence ou non d'une activité dactylographique. De plus, le temps imparti par le rédacteur à la mobilisation de ces processus peut être comptabilisé à la seconde près. Enfin, les protocoles d'allocation de ressources (c'est-à-dire les temps de réaction) sont liés à des comportements spécifiques de rédaction afin d'évaluer l'effort cognitif alloué aux différents processus.

\subsubsection{Procédure}

Le rédacteur réalise sa composition sur un ordinateur individuel (Levy et Ransdell, 1994). L'écran est divisé en panneaux permettant l'affichage de trois programmes tournant dans un environnement Windows. La partie gauche des trois quarts de l'écran propose une zone de rédaction faisant fonctionner un traitement de texte simplifié (contrôle du curseur, recherche et remplacement, option couper-coller) qui n'offre pas de possibilité de mise en forme ni de vérification orthographique et grammaticale (Traitement de texte NOTEPAD). Le panneau inférieur affiche une horloge qui permet de déterminer le moment où le rédacteur donne des réponses orales et/ou dactylographiées diverses. Le panneau supérieur droit contient la fenêtre de stimulation sonore gérée par un programme spécial (BEEPER), qui $1 /$ présente au hasard la sonde sonore, 2 / enregistre le temps de réaction du rédacteur lorsqu'il actionne la pédale pour répondre rapidement, et 3 / affiche le comptage des sondes proposées. Les modifications de l'écran sont aussi enregistrées ainsi que les pensées à haute voix du rédacteur.

Tout au long des sessions d'écriture, le participant est entraîné de façon progressive à réaliser la triple tâche (rédiger tout en verbalisant ses pensées et en réagissant à des sondes sonores). Dans une première session d'écriture, il rédige et réagit vite (les sondes étaient espacées de trente secondes plus ou moins quinze). Dans la session suivante, le participant est entraîné à la technique de protocoles verbaux concomitants en visionnant une bande vidéo de deux minutes qui explique son utilisation. Pour l'essentiel, il est encouragé à parler à voix haute pendant qu'il rédige, en verbalisant tout ce qui lui vient à l'esprit sans faire d'introspection ni justifier ce qu'il écrit ou comment il le fait. Pendant la condition expérimentale, l'expérimentateur incitera le rédacteur à « continuer à parler » chaque fois que s'installera un silence d'environ dix secondes. 
Chaque session de rédaction commence par une période de deux minutes pendant laquelle le participant s'entraîne à réagir rapidement. La moyenne de ces temps de réaction recueillis pendant les semaines successives de rédaction sera utilisée comme base pour calculer le temps de réaction pondéré de chaque rédacteur. Puis, le rédacteur reçoit la consigne pour composer pendant près de quarante minutes un texte (e.g. un article susceptible d'être soumis à l'éditeur d'un périodique national recherché).

\subsubsection{Variables dépendantes}

Les données sont dépouillées à l'aide du programme SIMULSCAN qui permet de rapprocher les différents événements enregistrés sur les canaux différents. Le programme EVENTLOG permet à des juges de repérer le début et la fin des enregistrements écrits et verbaux, de les catégoriser et de les rapprocher à la seconde près (cf. Levy et Ransdell, 1994 ; Ransdell, 1995). Le logiciel AGREE permet de vérifier l'accord entre les juges.

Les chercheurs disposent ainsi de données concernant la mobilisation des processus (déterminés à partir des protocoles verbaux et de l'activité dactylographique du rédacteur). Ces observations sont regroupées sous forme de matrices de transitions interprocessus pour une période de dix minutes. La fréquence des transitions entre les différents processus rédactionnels peut être repérée et visualisée ainsi que l'évolution de ce pattern tout au long de la session d'écriture et d'une session d'écriture à l'autre.

La mesure des temps de réaction pondérés est opérée. Elle est mise en relation avec la configuration des matrices de transitions.

Par ailleurs, des juges notent indépendamment chaque texte dactylographié selon I3 dimensions de qualité d'écriture regroupées ensuite pour établir une évaluation de sa qualité globale (échelle SSQS mise au point par Ransdell et Levy, 1996). Ces dimensions concernent le contenu, l'intention, le style, le choix de mots, l'organisation et les aspects de surface. 


\section{COMPARAISON DES DEUX PARADIGMES ET DE LEUR VALIDITÉ RESPECTIVE}

Le paradigme de Kellogg et celui de Levy et Ransdell constituent des variantes d'un même principe général: faire simultanément réaliser au rédacteur une triple tâche. Ces variantes imposent au rédacteur des contraintes de nature différente qui peuvent avoir des incidences sur la façon de rédiger et sur les caractéristiques linguistiques du texte alors produit. Ces variantes offrent-elles des «fenêtres » de qualité semblable sur les processus rédactionnels ainsi que sur leur coût ?

Les deux sous-parties qui suivent ont comme fonction d'évaluer, d'une part, la validité de la mesure de l'effort cognitif associé aux traitements opérés et, d'autre part, la réactivité (c'est-à-dire l'effet) du type de protocoles verbaux employés sur l'activité rédactionnelle.

\subsection{VALIDITÉ DE L'USAGE DES TEMPS DE RÉACTION}

Le paradigme de la triple tâche ayant comme fonction de mesurer les ressources attentionnelles (e.g. l'effort cognitif) associées à la réalisation de la tâche rédactionnelle, paraît, de prime abord, être de la famille du paradigme de la tâche ajoutée. En effet, afin d'évaluer l'effort cognitif, c'est-à-dire la quantité de ressources attentionnelles assignées à la réalisation d'une tâche, ou à celle de la mobilisation d'un processus ou d'un traitement particulier, les chercheurs utilisent depuis plus de vingt-cinq ans, le paradigme de la tâche ajoutée (Baddeley et Hitch, 1974; Kahneman, 1973 ; Kerr, 1973 ; Posner, 1978).

\subsection{Le principe du paradigme de la tâche ajoutée}

Dans le cadre du paradigme de la tâche ajoutée, le participant doit accomplir simultanément deux tâches. La réalisation conjointe de ces tâches exigera une quantité de ressources supé rieure à la capacité attentionnelle du participant. Pour gérer cette difficulté, le participant peut négliger la réalisation d'une des deux tâches afin de consommer ainsi moins de ressources. 
Pour imposer expérimentalement au participant une difficulté de ce type, l'expérimentateur peut lui demander de maintenir en mémoire un certain nombre d'items (mots, chiffres, configurations spatiales de points) durant l'exécution de la tâche principale. Il peut s'ensuivre une détérioration de la performance dans la tâche principale. Celle-ci est provoquée par l'effort cognitif lié à la réalisation de la tâche secondaire et révèle indirectement la quantité de ressources cognitives demandées par la tâche principale.

Cette technique de la tâche ajoutée ou double tâche, encore appelée tâche interférente ou concurrente, a fait l'objet de critiques méthodologiques (Fisk, Derrick et Schneider, 1986-1987) et de commentaires sur les interprétations fonctionnelles qu'elle pouvait ou non autoriser (Navon, 1984; Navon et Gopher, 1979 ; Pashler, 1984 ; Olive, 1997 ; Tricot et Chanquoy, 1996). Largement usitée en psychologie cognitive de l'attention (Camus, 1996), elle a été peu employée pour étudier l'évolution de l'effort cognitif associé à la production verbale orale (voir, par ex., Ford et Holmes, 1978 ; Power, 1985, 1986). Elle commence à peine à l'être en production verbale écrite. Compte tenu des options théoriques retenues par certains chercheurs (Gathercole et Baddeley, 1993 ; Just et Carpenter, 1992), l'usage de méthodes aptes à mesurer l'effort imparti à certains traitements linguistiques et/ou à la mobilisation des processus rédactionnels devrait être accentué.

L'expérience de Hupet, Schelstraete, Demaeght et Fayol (1996) exemplifie clairement comment les variantes d'une tâche concurrente (mémorisation d'une liste de mots vs opérations de calcul mental) sont utiles pour repérer, à propos des traitements orthographiques associés à la transcription de phrases, l'attribution de ressources attentionnelles aux différentes tâches. La charge de traitement imposée par la rétention de la liste de mots fait chuter la performance orthographique pour certains types de phrases comparativement à la condition sans charge. Toutefois, quel que soit le type de phrases à transcrire, le nombre de mots additionnels correctement rappelés reste identique (de 3 à 4 mots). Seule la performance de la tâche principale est détériorée. En revanche, lorsque la tâche ajoutée consiste à effectuer des opérations de calcul mental («véritable double tâche » selon Hupet et al., 1996), une relation entre les types de phrases donnés à dicter et le niveau d'exactitude du calcul atteint est mise 
en évidence, en même temps que l'orthographe se détériore. Dans ce cas-là, les participants opèrent des ajustements différents entre tâche principale et tâche secondaire. Ces différentes observations expérimentales permettent d'avancer que a) la nature de la tâche ajoutée est capitale car elle peut mobiliser ou non les différentes instances fonctionnelles de la mémoire de travail (en termes d'allocation de ressources et de traitement), et que $b$ ) les variations (ou non) de la performance dans la tâche secondaire sont aussi informatives que celles dans la tâche principale.

\subsubsection{La triple tâche, un paradigme non concurrent}

Comme l'a détaillé Olive (1997), à partir des critères de Fisk et al. (1986-1987), le paradigme de la triple tâche proposé par Kellogg (1987b) ainsi que Levy et Ransdell (1995) ne repose pas sur le principe d'une détérioration de la tâche principale par la réalisation des tâches ajoutées.

Pour évaluer le caractère non concurrent des tâches ajoutées « réagir vite » et « rétrospecter », plusieurs expériences à finalité méthodologique sont maintenant disponibles et ne permettent plus de tenir des propos « intuitifs » sur la non-validité de ce type de paradigme (Fayol, 1997 ; Richard, 1997).

Ainsi, la qualité des textes produits dans des situations de rédaction habituelle et non habituelle (tâche principale + tâches ajoutées) a été évaluée (Kellogg (1987a et b, 1988 ; Olive, 1997; Piolat, Roussey, Olive et Farioli, 1996). Le constat d'une absence de détérioration de la qualité des textes produits indique que les rédacteurs peuvent attribuer suffisamment de ressources à la gestion du texte afin de préserver les objectifs rédactionnels. Ils parviennent à réaliser un « compromis » dans la gestion des ressources attentionnelles en produisant un texte dont la qualité est préservée tout en réalisant la tâche secondaire.

De plus, si la réalisation des tâches ajoutées devait entrer en compétition importante avec celle de la tâche principale, il faudrait essayer d'en repérer l'impact sous forme de détérioration fonctionnelle. Par exemple, l'impact des tâches ajoutées pourrait être suffisamment important pour que, comparativement à ce qu'il fait en production libre, le rédacteur, trop contraint, soit obligé de minorer l'emploi des processus rédactionnels les plus 
coûteux (e.g. la planification et la révision). Il pourrait aussi, tout au long de son activité rédactionnelle, mobiliser les processus rédactionnels selon un pattern différent. Afin d'éprouver ces hypothèses, Piolat et al. (1996) ainsi qu'Olive (1997) ont évalué les effets de la tâche la plus concurrente : la cadence de distribution des sondes sonores auxquelles le rédacteur doit réagir rapidement en appuyant sur une presselle.

L'impact de la tâche ajoutée « Réagir vite » a été estimé en faisant varier la cadence de distribution des sondes sonores pendant la réalisation de la tâche principale. Trois cadences ont été imposées à trois groupes de rédacteurs. Le groupe à cadence rapide a subi une distribution des sondes sonores toutes les quinze secondes en moyenne dans un intervalle variant de dix à vingt secondes. La cadence moyenne utilisée par Kellogg (1987a) était ainsi divisée par deux. Le groupe à cadence « Kel$\log g »$ a reçu les sondes sonores toutes les trente secondes en moyenne dans un intervalle variant de quinze à quarante-cinq secondes. Le groupe à cadence lente était soumis à stimulation toutes les soixante secondes en moyenne dans un intervalle variant de quarante-cinq à soixante-quinze secondes.

Les principaux résultats montrent que la cadence avec laquelle les rédacteurs ont été interrompus influence significativement le temps de réaction. L'effort cognitif moyen des rédacteurs a été plus fort (502 ms) lorsque les sondes ont été distribuées le plus fréquemment mais aussi lorsqu'elles ont été délivrées selon la cadence la plus lente (525 ms). Ainsi, comparativement au groupe Kellogg $(399 \mathrm{~ms})$ à cadence intermédiaire de distribution des sondes, les résultats de ces deux groupes ont été du même ordre. Par ailleurs, les résultats concernant la mobilisation des processus tout au long de la rédaction sont étonnamment stables et sont conformes à ceux observés très fréquemment par Kellogg. Les variations de cadence ne remettent pas en cause le mode de gestion de l'ensemble de la tâche rédactionnelle : les rédacteurs mettent en texte plus fréquemment qu'ils ne planifient et ne révisent. De plus, au fur et à mesure de la composition, ils planifient de moins en moins alors qu'ils révisent de plus en plus. Enfin, la qualité des textes ne varie pas significativement en fonction des cadences. Ces différents résultats permettent de conclure qu'un compromis stratégique dans l'attribution des ressources entre les trois tâches seraient possible sans qu'il y ait une concurrence provo- 
quant une dégradation fonctionnelle significative de la tâche principale.

Une autre critique faite à cette méthode est qu'elle serait intrusive et modifierait le déroulement même de l'activité rédactionnelle. Dans l'expérience de Piolat et al. (1996), les rédacteurs ont, en effet, témoigné (sur une échelle en 7 points) d'une gêne importante en cadence de stimulation très rapide $(6,16)$, un peu moins intense en cadence Kellogg $(4,17)$ et atténuée en cadence lente (2,95 ; cf. Piolat et al., 1996). Toutefois, comme cela a été évoqué précédemment, ce sentiment d'intrusion ne transforme pas leur façon de mobiliser les processus rédactionnels, ni la qualité des textes qu'ils ont produits. Par ailleurs, la gêne ressentie par les rédacteurs en situation de triple tâche dont la réalisation dure au moins une demi-heure, pourrait provoquer une gestion irrégulière des ressources attentionnelles. En poursuivant l'analyse des données de la recherche de Piolat et al. (1996), Olive (1997) a montré que la durée des temps de réaction ne variait pas de façon significative entre le début et la fin de la rédaction. Ainsi, bien que contraints à réaliser de façon concomitante des tâches inhabituelles qui demandent beaucoup d'attention, les rédacteurs parviennent à maintenir un niveau de vigilance équivalent tout au long de la tâche (ils ne semblent pas non plus s'habituer à la réalisation de la triple tâche au point de répondre plus rapidement).

\subsubsection{Bilan}

Le fait de constater que les rédacteurs parviennent à négocier le coût de la cadence très rapide sans dégrader leur activité rédactionnelle ni la manière dont ils rédigent montre qu'ils ne sont pas en état de surcharge lorsqu'ils rédigent, contrairement à ce qui a été toujours affirmé depuis les propos de Hayes et Flower (I980). De plus, les résultats précédemment relatés indiquent que l'activité de rédaction n'est pas fonctionnellement bouleversée au point d'être dénaturée. Il est impropre de qualifier la tâche de réaction rapide d' « intempestive » comme le fait Richard (1997).

Enfin, selon les objectifs des recherches à entreprendre, la cadence de distribution des signaux sonores doit être volontairement choisie (Piolat, Olive, Roussey, Thunin et Ziegler, sous presse). Par exemple, les rédacteurs novices ou peu expérimentés 
devraient être testés avec une cadence de distribution des signaux plus lente afin de réaliser leur tâche principale dans des conditions aussi peu intrusives que celles proposées jusqu'à maintenant aux adultes par Kellogg.

\subsection{VALIDITÉ DE L'USAGE DES PROTOCOLES VERBAUX}

Malgré les critiques dont elle fait encore l'objet, la méthode des protocoles verbaux est toujours largement utilisée, depuis plus de vingt ans, pour étudier les processus et les connaissances engagés dans les activités cognitives (Ericsson et Simon, 1993 ; Russo, Johnson et Stephens, 1989; Wilson, 1994). A partir des travaux princeps de Hayes et Flower (1980; voir aussi Swarts, Flower et Hayes, 1984 ; Hayes et Flower, 1983 ; Hayes, Flower, Schriver, Stratman et Carey, 1987), elle l'est aussi, par exemple, pour étudier la rédaction de textes en langue maternelle (Smagorinsky, 1994) ainsi qu'en langue seconde (Cumming, 1994). Elle peut aussi aider à l'analyse de traitements rédactionnels plus spécifiques comme les stratégies orthographiques (Varnhagen, 1995). Cette méthode autorise de plus une mise en relation de la mobilisation des processus rédactionnels et la qualité du texte produit conjointement (Breetvelt, van den Bergh et Rijlaarsdam, 1994, 1996). Enfin, l'analyse simultanée des pauses et des retranscriptions des pensées émises à haute voix pendant la composition a ouvert une voie nettement bénéfique à l'identification des processus et connaissances en oeuvre chez les rédacteurs adultes (Hayes et Flower, 1980; Kaufer, Hayes et Flower, 1986) et les rédacteurs novices (Burtis, Bereiter, Scardamalia et Tetroe, 1983). Les paradigmes de Kellogg (1987) et de Levy et Ransdell (1995) y recourent, tous deux. Il est nécessaire d'en évaluer les avantages et inconvénients méthodologiques.

La méthode des protocoles verbaux comporte des variantes qui ont été classées en fonction du moment où la verbalisation est sollicitée par rapport à la composition du texte et en fonction du type de consigne donné au rédacteur. Plusieurs questions concernant la validité de ces variantes ont été posées (Cooper, 1983 ; Cooper et Holzman, 1983, 1985) et le sont toujours (Fayol, 1997 ; Gufoni, 1996). La verbalisation concomitante de pensées à haute voix n'affecte-t-elle pas la nature et la mobilisation des processus rédactionnels? Les protocoles rétrospectifs 
(compte rendu a posteriori des pensées) ne sont-ils pas qu'un souvenir imprécis et reconstruit de l'activité mentale que le rédacteur a développée pendant sa composition?

Lorsque les chercheurs ont répondu intuitivement à ces deux questions sans faire mention des travaux ayant mis à l'épreuve la réactivité de ces méthodes, ils rejettent généralement plus vivement la variante de procédure de Kellogg (1987b) que celle de Levy et Ransdell (1994). La procédure de ce premier auteur cumulerait, selon eux, une double difficulté la transmission des pensées à haute voix est différée et réalisée sous forme de catégorisation.

\subsubsection{Verbalisations concomitantes ou différées ?}

Le caractère concomitant ou différé de la verbalisation que le rédacteur doit réaliser a-t-il d'importantes conséquences sur la qualité des informations obtenues?

Levy et Ransdell (1995) préconisent d'utiliser la méthode des protocoles concomitants (simultanés) qui demande au rédacteur de penser à haute voix tandis qu'il compose. Afin de soutenir cette recommandation, ils ont comparé les résultats ainsi obtenus à ceux recueillis avec la méthode des protocoles rétrospectifs qui impose aux rédacteurs de verbaliser leurs pensées développées au cours de la rédaction, une fois le texte achevé, en visionnant leur activité rédactionnelle. Cette seconde variante avait été mise au point par Schumacher, Klare, Cronin et Moses (1984) pour éviter au rédacteur de dénaturer son activité rédactionnelle en réalisant une double tâche (rédiger et verbaliser à haute voix ses pensées).

La première critique adressée à l'usage des protocoles rétrospectifs est que le fait de différer la verbalisation provoquerait des phénomènes d'oubli et de reconstruction plus ou moins importants. L'ampleur de ces phénomènes dépendrait des conditions dans lesquelles la rétrospection est utilisée, comme, par exemple, la durée du délai entre la fin de la rédaction et le début de la rétrospection (Ericsson et Simon, 1993 ; Gufoni, 1996) ou la longueur des textes à rédiger (Gufoni, 1996).

Pour leur part, Levy et Ransdell (1995) ainsi que Levy, Marek et Lea (1996) ont comparé le contenu des protocoles concomitants et rétrospectifs de rédacteurs. Levy et al. (1996) ont constaté que les rédacteurs sont incapables de se rappeler 
avec précision (même lorsque leur certitude dans leur étiquetage est élevée) les processus qu'ils ont mobilisés pendant leur session de rédaction (60\% des classements rétrospectifs sont concordants dans les meilleurs des cas et seulement $10 \%$ dans les pires des cas).

Ces résultats inviteraient à préférer le paradigme de Levy et Ransdell (1995) à celui de Kellogg (1987b). Toutefois, il est essentiel de noter que même si Kellogg (1987b, 1988) qualifie de « rétrospective » la catégorisation qu'il impose au rédacteur, celle-ci n'est pas faite une fois le texte achevé, mais immédiatement après chaque réaction rapide qui est réalisée toutes les trente secondes environ pendant la rédaction. Le rédacteur rend compte de son activité mentale une à trois secondes seulement après avoir été interrompu par le signal sonore. Dans ce contexte de catégorisation concomitante (appelée sans doute à tort rétrospective), il n'est pas pertinent d'imputer à la méthode de Kellogg les biais méthodologiques du type de ceux observés par Levy et Ransdell (I995) ainsi que Levy, Marek et Lea (I996). A ce jour, aucune donnée expérimentale ne permet de conclure à des oublis ainsi qu'à une activité de reconstruction de la part du rédacteur lorsqu'il catégorise ce à quoi il était en train de penser juste après avoir été interrompu pour réagir vite.

\subsubsection{Verbalisations libres ou dirigées}

Un des points majeurs sur lesquels le paradigme de Kellogg (1987) et celui de Levy et Ransdell (1995) diffèrent, concerne la façon dont le rédacteur rend compte de son activité mentale. En effet, la consigne donnée par Levy et Ransdell (1995) incite le rédacteur à dire librement tout ce qui lui vient à l'esprit tandis qu'il rédige son texte. À l'opposé, la consigne de Kellogg (1987) exige du rédacteur qu'il catégorise brièvement ce à quoi il pensait.

La consigne donnée aux rédacteurs pour verbaliser à haute voix transforme-t-elle l'activité rédactionnelle ? Ericsson et Simon (1993) ont recensé les différents types de verbalisation provoqués par diverses consignes. Ils différencient les verbalisations, simple vocalisation de l'articulation intérieure (niveau I) des verbalisations qui impliquent une activité de description du contenu de la pensée comme peut le faire un étiquetage (niveau II). Ils citent, enfin, les verbalisations qui impliquent 
d'expliquer (niveau III). Un bilan opéré sur de nombreuses expérimentations leur permet de conclure que les verbalisations additionnelles de niveau I et II ne changent pas la nature des processus engagés dans la tâche principale mais en ralentissent la réalisation. Celles de niveau III peuvent, en revanche, perturber le cours de la réalisation de l'activité principale car elles imposent un surcroît trop important d'attention.

Levy et Ransdell (1995) imposent à leurs rédacteurs des verbalisations de niveau I. La catégorisation imposée par Kellogg (1987) à ses rédacteurs ne peut être comparée à une « explication » de niveau III. Comme la variante utilisée par Levy et Ransdell (1995), celle employée par Kellogg (1987) imposerait aux rédacteurs une utilisation des informations présentes dans la mémoire de travail. Elle ne peut, ainsi, provoquer de changement majeur dans la mobilisation des processus rédactionnels. Une part des observations faites par Gufoni (1996) qui a comparé le contenu des informations rétrospectives données par des rédacteurs de façon dirigée ou non, soutiendrait cette interprétation. Elle souligne que leur choix de la rétrospection dirigée ou non dépend de la finalité plus ou moins exploratoire de la recherche.

Dans le cadre de ce débat, Levy et Ransdell (1995) estiment que lorsque l'on donne aux rédacteurs la possibilité de témoigner sur leur façon de rédiger, ils utilisent leurs conceptions sur la façon dont un rédacteur doit opérer. De ce fait, la rétrospection dirigée pourrait fonctionner comme une sorte de témoignage sur soi et permettre de rendre compte non pas de ce que fait réellement le rédacteur mais de ce qu'il pense qu'il doit faire. Aussi, ces auteurs ont comparé si les rédacteurs allouent du temps (et de l'effort) aux processus rédactionnels comme ils disent le faire. Pour cela, les rédacteurs ont évalué (en \%) le temps qu'ils pensent avoir passé à planifier, à rédiger, à se relire et à se corriger. Ils ont aussi estimé sur une échelle en sept points la quantité d'effort que la réalisation de ces différentes activités leur a demandée.

Les principaux résultats montrent que les rédacteurs prévoient plus précisément la quantité de temps et d'effort qu'ils alloueraient à la planification et à la mise en texte qu'ils ne le font pour le réexamen et la révision. Les participants surestimaient fortement le temps passé au réexamen et à la révision. Afin de critiquer Kellogg (1987b), Levy et Ransdell (1995) souli- 
gnent que ces témoignages sur la mobilisation des processus sont convergents avec les rétrospections observées par Kellogg (1987b) et qu'ils coïncident de façon plus étroite avec ces rétrospections qu'ils ne le font avec les comportements rédactionnels qui leurs sont associés (activité concrètement réalisée au clavier). Ils considèrent, alors, que la rétrospection dirigée reflète partiellement les conceptions des participants. Levy et Ransdell (1995) situent la rétrospection dirigée entre les témoignages sur soi et les données de protocoles verbaux quand il s'agit de rendre compte de façon adéquate des comportements réels de rédaction.

Pour sa part Kellogg (1987b) a comparé les classifications de protocoles verbaux faites par des rédacteurs et des juges entraînés et conclut à un accord satisfaisant. Il souligne la capacité des rédacteurs adultes à utiliser correctement la catégorisation des processus. Il en infère que la capacité des rédacteurs à catégoriser en temps réel leur activité doit être tout aussi valide.

La catégorisation des pensées mentales, comparativement à leur expression librement réalisée par le rédacteur, fait l'objet d'une autre critique. Pour Levy et Ransdell (1995), la quantité d'informations disponibles lorsque les rédacteurs verbalisent librement est plus importante et détaillée ainsi que plus continue. Kellogg (1987) ne fait que «sonder», toutes les trente secondes, l'activité mentale des rédacteurs. Il faut toutefois noter que le souci du détail fonctionnel (processus et sousprocessus évoqués librement par les rédacteurs) n'est plus de mise lorsque Levy et Ransdell (1994, 1995) présentent leurs résultats. Ces auteurs regroupent leurs résultats en quatre rubriques (Planifier, Mettre en texte, Évaluer et Réviser) et, compte tenu de leurs observations, rassemblent même en un seul processus évaluer et réviser. La mise en évidence des «signatures » des rédacteurs est faite selon une échelle de temps de l'ordre de dix minutes. La richesse des données obtenues pendant plus d'une demi-heure d'émission de protocoles verbaux ininterrompus paraît ainsi peu cruciale, alors même que le dépouillement des protocoles a dû être particulièrement long (cf. $§ 1.2 .2$ ).

Le nombre des catégories données au rédacteur pour qualifier son activité mentale peut être problématique. En s'inspirant de la classification de Schumacher, Klare, Cronin et Moses (1984), qui comporte une vingtaine de catégories, Levy, Lea et Marek (1996) ont montré que les rédacteurs avaient des diffi- 
cultés à employer un tel éventail de catégories. Selon eux, la mémorisation d'un tel éventail, afin d'opérer la catégorisation demandée, dépasse largement les possibilités de la mémoire de travail des rédacteurs et rend peu valide l'utilisation des protocoles rétrospectifs. Kellogg (1987), qui invite les rédacteurs à employer seulement un petit nombre de catégories (4 en général : Planifier, Mettre en texte, Réviser et Autre), ne peut être soumis à cette critique.

Il est. essentiel toutefois de s'assurer de la maîtrise de ces catégories par le rédacteur. Barbier, Piolat et Roussey (1998) ont, en effet, constaté que des élèves âgés de 15-16 ans apprennent avec difficulté à étiqueter leurs traitements ( « Je fais attention à l'Orthographe, au Lexique, à la Grammaire, aux Idées, à l'Organisation du Texte ou Autre ») lors de la tâche d'entraînement. De plus, lors de la réalisation de la tâche de rétrospection, ces élèves choisissent assez souvent l'étiquette «Autre », alternative indiquant leur difficulté à identifier ce qu'ils faisaient en écrivant. Ainsi, l'expérimentateur qui souhaite utiliser la mise au jour des pensées des rédacteurs par catégorisation doit, d'une part, pouvoir choisir le nombre et la dénomination des processus qu'il va proposer aux participants (voir Piolat et al., sous presse). Le chercheur doit, d'autre part, s'assurer, via un entraînement suffisant, que les participants pourront les employer avec discernement. Il peut, enfin, se fixer comme objectif expérimental de rechercher ce que des individus de niveaux d'expertise rédactionnelle divers parviennent à dire - via cette catégorisation « en temps réel » - sur certains de leurs traitements.

Dans le même ordre d'idée, en voulant s'assurer de l'effet du niveau de maîtrise de la capacité à catégoriser ses pensées, Piolat et al. (1996) ont montré que, comparativement à l'entraînement habituellement réalisé par Kellogg, un apprentissage plus approfondi de l'activité de catégorisation des processus ne transforme pas significativement leur mobilisation tout au long de l'activité de composition de texte. En revanche, le temps de réaction pondéré moyen des rédacteurs plus entraînés à réaliser une tâche de rétrospection est plus long que celui des rédacteurs entraînés selon les consignes de Kellogg. Toutefois, ces rédacteurs catégorisent (temps mis pour désigner un processus) leur activité de façon plus rapide que le groupe moins entraîné. Tout se passe comme si les rédacteurs les plus entraînés utilisaient des ressour- 
ces attentionnelles pour savoir ce qu'ils font au moment même où ils rédigent, gagnant ainsi un peu de temps lors de la désignation du processus. Ainsi, les participants pourraient être conduits à gérer stratégiquement la triple tâche selon la façon dont ils ont été entraînés et informés. Face à ce type d'observations, certains chercheurs concluront à la mise en place d'un nécessaire compromis par le rédacteur entre les trois tâches (rédiger, réagir vite et catégoriser) ; d'autres (en écho aux objections de Fisk, Derrick et Schneider, 1986-1987) évoqueront une « contamination » méthodologiquement critiquable entre les trois tâches. De nouvelles expérimentations doivent être faites pour mieux comprendre ces phénomènes.

Enfin, les paradigmes de Kellogg et de Levy et Ransdell prêtent le flanc à la critique commune selon laquelle les informations verbalisables sont celles sur lesquelles l'attention est focalisée (donc accessibles parce que non automatisées ; Ericsson et Simon, 1993). Les détracteurs de la méthode des protocoles verbaux en tirent deux arguments. Le premier consiste à affirmer que la méthode favorisait la verbalisation par le rédacteur de phénomènes (et même d'épiphénomènes) qui, lors de son activité, retiennent particulièrement son attention (e.g. difficulté lexicale ou syntaxique). La régularité des configurations de processus observés par Levy et Ransdell (I995) au travers d'une douzaine de sessions d'écriture par rédacteurs permet de rejeter une telle critique. Le second argument concerne le fait que les processus dont le rédacteur peut parler sont forcément conscients, les autres automatiques restant inaccessibles. En étudiant de la production verbale orale, les chercheurs modélisent des particularités fonctionnelles clairement automatiques (e.g., pour les opérations réalisées par le «formulateur », cf. Levelt, 1989). Une étude comparable doit être conduite dans le champ de la rédaction de texte. Mais l'analyse de la production verbale écrite implique, aussi, la mise au jour de processus mobilisés de façon clairement délibérée par le rédacteur. Étudier les processus consciemment activés constitue un enjeu déjà fort complexe. L'analyse de l'intervention de processus automatisés comme ceux de la transcription graphique ou la sélection lexicale ne pourra être faite via cette méthode. Mais ce n'est pas, bien sûr, ce qui est recherché par les utilisateurs de la méthode de la triple tâche pour repérer certaines caractéristiques fonctionnelles de la rédaction de textes. 


\subsubsection{La réactivité de la verbalisation}

Une des critiques la plus fréquemment adressées à la méthode des protocoles verbaux depuis une vingtaine d'années est que l'imposition de cette tâche additionnelle influence la réa lisation de la tâche principale et pourrait même la dénaturer. L'évaluation de la « réactivité » des pensées à haute voix sur l'activité principale est d'ailleurs encore entreprise. Ce qui est mis en évidence par les expérimentations faites à ce sujet est que ce sont surtout certaines caractéristiques temporelles de l'activité qui sont modifiées (Ericsson et Simon, 1993).

Ainsi, Stratman et Hamps-Lyons (1994) ont envisagé les conséquences de la verbalisation sur la capacité à détecter et à réviser différents types d'erreurs inclus dans un texte. Ils ont constaté que la verbalisation affectait plus la fréquence d'activation des sous-processus de révision que leur nature.

Ransdell (1990, 1995) a, pour sa part, observé que, lorsque les rédacteurs verbalisent à haute voix leurs pensées de façon concomitante, ils ralentissent leur activité de composition en produisant moins de mots et de propositions par minute que lorsqu'ils rédigent en silence. Toutefois, le volume verbal global ainsi que la complexité syntaxique ne sont pas affectés. Levy et Ransdell (1995) se sont, quant à eux, donné comme objectif de repérer si l'impact des verbalisations à haute voix était du même ordre sur plusieurs sessions successives de rédaction. Ils observent que les tâches ajoutées (réagir vite et verbaliser à haute voix ses pensées) n'affectent pas significativement la stabilité de la répartition du temps et de l'effort chez le rédacteur. Le ralentissement du débit rédactionnel est, au plus, transitoire ; les rédacteurs sont rapidement capables de rédiger de façon efficace, même en produisant des protocoles. La rédaction n'est pas massivement transformée par les protocoles verbaux concomitants car ceux-ci impliquent principalement de coder et décoder verbalement une information disponible en mémoire de travail.

Janssen, van Waes et van den Bergh (1996) ont étudié la durée et la fréquence des pauses produites par des rédacteurs qui utilisent un traitement de texte lorsqu'ils verbalisent ou non leurs pensées à haute voix. Ils retrouvent le résultat selon lequel la tâche additionnelle implique un allongement de la durée des pauses. Ils constatent, aussi, que l'impact de cette tâche additionnelle est en interaction avec les exigences de la tâche princi- 
pale. En effet, la variation des pauses dépend aussi des types de textes à composer qui, provoquant eux-mêmes des stratégies de récupération et d'organisation de connaissances différentes, influencent donc la durée et la fréquence des pauses. De plus, les effets de la tâche additionnelle seraient différents selon les types de rédacteurs.

A l'issue de ses différentes recherches, il est possible de conclure provisoirement à une faible réactivité de la verbalisation à haute voix qui ne transforme pas de façon notable la rédaction (Ransdell, 1990, 1995 ; Levy et Ransdell, 1995).

\subsection{BILAN}

En résumé, compte tenu des résultats et des données obtenus, il est impossible d'invoquer une dégradation massive de la réalisation de la tâche rédactionnelle, lorsque les individus verbalisent leurs pensées pendant ou après, de façon dirigée ou non (Fayol, 1997). Le faire consisterait à se priver d'informations particulièrement intéressantes alors même que les apports technologiques permettent de mieux saisir et de mieux analyser ces observables.

Levy et Ransdell (1995) considèrent, en revanche, qu'il n'est pas possible de se contenter de la seule utilisation des protocoles verbaux pour étudier la rédaction de textes comme le proposaient Hayes et Flower (1983). Ils trouvent indispensable de rapprocher les protocoles écrits (enregistrements en temps réel de la rédaction réalisée avec un traitement de texte) des protocoles verbaux (ce que les rédacteurs disent pendant qu'ils écrivent) pour établir un témoignage convergent concernant l'analyse du temps passé à rédiger. Le recueil des protocoles d'allocation de ressources (temps de réaction d'interférence, liés à des comportements spécifiques de rédaction) permettrait alors de catégoriser l'effort cognitif alloué aux processus. Autrement dit, les protocoles écrits et verbaux facilitent la définition opérationnelle des processus basiques (planification, mise en texte, réexamen et révision). Les protocoles écrits fournissent l'enregistrement de l'élaboration et des corrections, apportant ainsi plus d'informations que le simple produit écrit final. Leur rapprochement permet de statuer sur la signification des pauses (planification ou réexamen). 
Comparativement, la méthode employée par Kellogg (19876) ne favorise pas une vision aussi intégrée du fonctionnement du rédacteur via les trois fenêtres cognitives que constituent les protocoles écrits, les protocoles verbaux, et les protocoles d'allocation de ressources puisque seuls les deux derniers types de protocoles sont disponibles. Toutefois, le fait que les rédacteurs ne soient pas obligés d'utiliser un traitement de texte offre par défaut d'autres possibilités de recherche (cf. Piolat et al., 1999). Les populations de rédacteurs peuvent être, par exemple, des rédacteurs novices très jeunes et ne sont pas réduites aux seuls utilisateurs de traitement de texte. Il faut, en outre, bien noter que l'usage d'un traitement de texte transforme les stratégies rédactionnelles mises en place avec l'usage du papiercrayon, particulièrement les procédures de planification et de révision (Piolat et Blaye, 1991 ; Piolat, Isnard et Della Valle, 1993). Levy et Ransdell (1995) ont analysé quasi exclusivement les activités rédactionnelles d'étudiants pour lesquels l'usage du traitement de texte est familier.

Une critique d'une tout autre nature peut être adressée à la consigne selon laquelle les rédacteurs doivent « désigner le processus le plus important » (cf. Kellogg, 19876). Cette consigne inciterait le rédacteur à procéder à une catégorisation de son activité et de ses pensées à l'aide d'un seul processus (soit il planifiait, soit il mettait en texte, soit il révisait). Ainsi, le paradigme contraindrait le rédacteur à donner une image de son fonctionnement contestable : l'activation des processus rédactionnels serait opérée de façon récursive et sérielle. Dans le cadre des études en production verbale orale, cette conception a été critiquée (Ford et Holmes, 1978; Kempen et Hoenkamp, 1987). Plus récemment, Kellogg $(1994,1996)$ lui-même indique que le rédacteur peut activer des pensées plus complexes en raison de sa capacité à mobiliser plusieurs processus en parallèle. En étudiant les pauses à l'écrit, Foulin (1995) lui aussi considère que certains processus sont activés simultanément. Pour leur part, Levy et Ransdell (1995) le constatent pour $8 \%$ des informations issues de leurs protocoles concomitants. Aussi, Pélissier et Piolat (1998) ont comparé les informations issues d'une activité de rétrospection imposant une catégorisation de l'activité en cours (paradigme standard de Kellogg, 19876) à celles issues d'une activité de rétrospection laissant le rédacteur libre de désigner un ou deux processus quand il a été interrompu par les signaux 
sonores. Les principaux résultats montrent que le pattern de mobilisation des processus n'est pas remis en cause par cette dernière possibilité et que si les processus de «traduction » et d' « exécution » (qui correspondent à la « mise en texte ») sont souvent désignés de façon associée, certaines associations ne sont pas produites par les rédacteurs (par ex., «Planifier» et « Réviser »). L'activation en parallèle de processus par les rédacteurs serait limitée à une seule association.

Au total, les ajustements des rédacteurs aux conditions expérimentales imposées à l'aide d'une modulation de leur effort cognitif (tel qu'il est mesuré par la tâche ajoutée), l'absence de transformation de la stratégie rédactionnelle d'ensemble (traduite par le pattern de mobilisation des processus dans les trois phases de composition) ainsi que l'absence de variation des indicateurs concernant la performance et la qualité des textes permettent de conclure que les rédacteurs soumis au paradigme de la triple tâche ne sont pas en état de « surcharge » cognitive. Autrement dit, malgré l'ajout des tâches de réaction et de rétrospection, tout se passe comme si les rédacteurs pouvaient encore contrôler de façon intentionnelle le déroulement de leur activité principale (rédiger) tout en préservant leur objectif : atteindre une certaine qualité textuelle.

\section{LES PRINCIPAUX ACQUIS EXPÉRIMENTAUX}

Alors qu'ils utilisent un paradigme de recherche comparable, Kellogg (1994) et Levy et Ransdell (1995) ne se sont pas fixé les mêmes objectifs. Pour l'essentiel, le programme de recherches de Kellogg a concerné jusqu'à ce jour l'effet des caractéristiques endogènes du rédacteur (e.g. niveau de connaissances) ou des conditions de production (e.g. tâches de préécriture imposées ou médium de production) sur l'effort cognitif et la mobilisation des processus rédactionnels. Le programme de recherches de Levy et Ransdell (jusqu'à la publication de Levy, 1997) a consisté à mettre en évidence la diversité des stratégies rédactionnelles individuelles ainsi que leur stabilité dans le temps chez un même rédacteur. Pour ces deux équipes de recherche, la mise en relation du fonctionnement du rédacteur et de la qualité du texte qu'il produit reste une préoccupation constante. Les données 
rassemblées ci-après sont présentées de façon très succincte, sans les étayages théoriques qui sont disponibles dans les publications. Cette rapide présentation a comme fonction principale d'illustrer les types de constats réalisés grâce au paradigme de la triple tâche.

\subsection{MISES EN ÉVIDENCE DES CARACTÉRISTIQUES FONCTIONNELLES GÉNÉRALES DU RÉDACTEUR}

Avec le paradigme de la triple tâche, peut-on repérer des « invariants » dans le coût des processus rédactionnels et dans les façons de procéder des rédacteurs ? Autrement dit, est-il possible de dégager des invariants interindividuels mais aussi des profils types de rédacteurs?

\subsubsection{Invariants interindividuels durant une session de production}

La mise en évidence d'un fonctionnement rédactionnel basique au cours d'une session de rédaction peut être abordée selon une double perspective

a) Y a-t-il un (ou des) processus rédactionnel(s) dont le coût ou la mobilisation dominent les autres ?

b) Le coût et la mobilisation de chacun des processus rédactionnels évoluent-ils tout au long d'une session de rédaction?

Kellogg (1987b, 1988, exp. 1) a montré que l'effort cognitif associé à la planification et à la révision $(360 \mathrm{~ms})$ était le plus élevé comparativement à celui de la mise en texte. Ce résultat a été répliqué dans certaines recherches (Olive, Piolat et Polge, 1997) mais pas dans d'autres (Kellogg, I988, exp. 2 ; Kellog, 1993 ; Kellogg et Mueller, 1993).

Pour leur part, Levy et Ransdell (1995) ont montré que la planification et la mise en texte requièrent un effort substantiel et que cet effort est plus important que celui dévolu au réexa men et à la révision. Dans des situations expérimentales reprenant le paradigme expérimental de Kellogg (1987b), Piolat, Roussey, Olive et Farioli (1996) ainsi que Olive, Piolat et Roussey ( $1997 a$ et $b$ ) ont montré que seule la planification demandait plus d'effort. Penningroth et Rosenberg (1995) ont eux aussi mis 
en évidence le coût supérieur du seul processus de planification comparativement à celui de mise en texte mais pas à celui de révision. Enfin, en utilisant le paradigme de Kellogg, Piolat, Roussey et Rous (1996) n'ont pas observé de différence significative entre le coût des trois processus rédactionnels.

Ainsi, une majorité des résultats obtenus avec le paradigme de la triple tâche permet d'avancer que la planification serait plus coûteuse que le processus de mise en texte. Le coût du processus de révision serait quant à lui plutôt supérieur à celui de la mise en texte. Le degré d'automatisation plus important de ce dernier processus est d'ailleurs souvent évoqué pour expliquer ce moindre coût ; la planification et la révision nécessitant des traitements fortement délibérés seraient donc plus coûteuses (Fayol, I997 ; Kellogg, 1996 ; Levy, 1997 ; McCutchen, 1996).

Il faut se demander si l'effort cognitif associé aux processus rédactionnels peut évoluer (en diminution comme en augmentation) tout au long de la session d'écriture. L'observation de résultats significatifs en faveur d'une évolution des temps de réaction tout au long de la session d'écriture autoriserait aussi à envisager de possibles effets de répercussion des traitements les uns sur les autres. Autrement dit, le coût lié à la mobilisation de chacun des processus pourrait évoluer au fur et à mesure que le rédacteur avance dans la composition de son texte. Par exemple, le coût élevé de la planification pourrait baisser au fur et à mesure que le rédacteur cerne mieux, compte tenu de ce qu'il a déjà réalisé, le contenu qui lui reste à programmer (ce type de phénomène a été mis en évidence par l'étude des pauses, cf. Foulin, 1995). Les utilisateurs de la triple tâche n'ont pas analysé leurs résultats dans ce sens. Cette piste de recherche reste à explorer.

Kellogg (1987b, I988) a montré que la mise en texte est le processus le plus activé (environ la moitié du temps de composition) ; la mobilisation de la planification serait de l'ordre de $30 \%$ et celle de la révision de $25 \%$. Les résultats des recherches de Olive, Piolat et Polge (I997), ainsi que Piolat, Roussey, Olive et Farioli (1996) sont du même ordre.

Pour leur part, Levy et Ransdell (1995) ont trouvé des proportions de grandeur comparables sauf pour les processus de contrôle de la rédaction dont la mobilisation est moins fréquente $($ Planification $=40 \%$; Mise en texte $=45 \%$; Réexa- 
men $=8 \%$; Révision $=6 \%)$. Penningroth et Rosenberg $(1995)$ ou encore Piolat, Roussey et Rous (1996) ont obtenu, quant à eux, une mobilisation de la mise en texte comparable (44\%) mais, en revanche, une fréquence faible de planification (10\%) contre une fréquence importante de révision $(40 \%)$.

Ainsi, dans la majorité des recherches, les rédacteurs mobilisent nettement la mise en texte. Mais ce sont les processus de planification et révision dont la mobilisation serait sensible aux effets des types de contextes rédactionnels qui seront évoqués plus bas.

Pour mettre en évidence l'évolution de la mobilisation des différents processus rédactionnels tout au long de l'activité, Kellogg (1994) a divisé en trois la durée de composition totale de chaque rédacteur. Il a comparé, à l'intérieur de ces trois phases, la fréquence de mobilisation des trois processus. Les résultats présentés dans la quasi-totalité de ses publications permettent de conclure que la mise en texte est le processus le plus activé pendant les trois phases rédactionnelles. En revanche, la planification est de moins en moins mobilisée de la première à la dernière phase et inversement pour la révision. Ce pattern de résultats est aussi obtenu par Penningroth et Rosenberg (1995) ainsi que Piolat, Roussey, Olive et Farioli (I996).

Levy et Ransdell (1995) ont procédé à des analyses plus minutieuses (de cinq minutes en cinq minutes) de l'évolution de la mobilisation des processus rédactionnels mais leurs résultats sont compatibles avec ceux des auteurs précédents. Vers les deux tiers de la durée d'une session de rédaction (d'environ quarante minutes), les rédacteurs passent d'une mobilisation très fréquente de la planification à celle de la mise en texte $(85 \%)$. $\grave{A}$ partir de ce moment, le temps de mise en texte chute légèrement. La révision qui occupait seulement 2 à $3 \%$ du temps des rédacteurs environ jusqu'à la mi-parcours d'une session occupe plus de $10 \%$ du temps de rédaction vers la fin de la session. Le temps de réexamen est réparti de façon similaire, mais il commence à augmenter plus tôt, dès le premier quart de chaque session.

Il est, maintenant, indispensable de s'assurer que l'allocation de temps et l'effort alloué aux processus rédactionnels clairement repérés lors de la production d'un texte restent stables tout au long de sessions de production réalisées dans des contextes comparables. 


\section{I.2. La stabilité fonctionnelle \\ des rédacteurs au cours des sessions}

Levy et Ransdell (1995) ont étudié le fonctionnement d'une dizaine de rédacteurs pendant 10 sessions d'écriture (situations de communications comparables : type de texte, enjeux, destina taire, etc.). Leurs résultats (matrices de probabilités de transition entre les différents processus rédactionnels de dix en dix minutes) montrent que, pour chaque rédacteur, la configuration d'allocation de temps aux sous-processus est constante au cours des sessions successives de rédaction. Ces auteurs soulignent aussi que la durée des temps de réaction est « remarquablement stable ».

Ces résultats ont des conséquences méthodologiques importantes pour la recherche dans ce domaine. L'activité rédactionnelle fait partie de tâches cognitives complexes dont l'étude s'avère particulièrement longue et lourde pour le chercheur. La stratégie rédactionnelle d'un individu serait suffisamment stable dans un type de situation de production donné (ce dernier devant être clairement caractérisé) pour éviter au chercheur d'enregistrer plusieurs sessions de rédaction.

\subsection{EFFETS DES CARACTÉRISTIQUES DES RÉDACTEURS}

Avec le paradigme de la triple tâche, des invariants fonctionnels ont pu être repérés. Toutefois, en utilisant d'autres méthodes, les chercheurs ont fait état de fortes variations interindivi duelles. Les études des effets de différentes caractéristiques des rédacteurs (connaissances, habileté linguistique, etc.) sur leur façon de procéder, autrement dit leur stratégie rédactionnelle, sont rapportées ci-après.

\subsubsection{Effet des connaissances et des habiletés linguistiques}

Dès ses premières expérimentations, Kellogg (1987b) a montré l'importance des connaissances thématiques des rédacteurs sur les caractéristiques fonctionnelles de leur activité. L'effort cognitif fourni par les rédacteurs disposant de peu de connaissances est toujours plus important que celui fourni par des rédacteurs possédant plus de connaissances. En revanche, la configuration de la mobilisation d'ensemble des processus ainsi que l'évolution de l'appel à ces processus tout au long de la ses- 
sion ne sont pas modifiées par le niveau de connaissances possédé par les rédacteurs (Kellogg, 1987a).

Penningroth et Rosenberg (1995) ont, pour leur part, contraint les rédacteurs à intégrer dans leur récit une conclusion comportant soit peu d'informations (niveau de charge de traitement bas), soit comportant plusieurs informations (niveau de charge de traitement élevé). Cette seconde condition n'a pas contraint les rédacteurs à fournir plus d'effort lors de la production du récit, mais, en revanche, les a incité à mobiliser différemment leurs processus rédactionnels. Cependant, le statut de cette charge de traitement peut être questionné : les conditions expérimentales provoquent-elles surtout une mobilisation plus ou moins importante de connaissances thématiques, ou bien imposent-elles seulement une différence de régulation verbale du récit, faisant appel aux habiletés linguistiques des rédacteurs ?

Kellogg (1993, I994) a mesuré le niveau d'habileté linguistique des rédacteurs à l'aide d'un test (ACT English subtest) mais n'a pas observé d'effet de ce facteur sur l'effort cognitif déve loppé par les rédacteurs, ni sur la mobilisation des processus. Olive, Piolat et Roussey (1997a et b) ont reproduit la seconde expérience de Kellogg (1987b) concernant l'impact du niveau de connaissances des rédacteurs tout en contrôlant leur niveau d'habileté linguistique. Ils n'ont pas dupliqué les résultats de Kellogg (1987b, 1993, 1994). L'effort cognitif que développent les rédacteurs selon leur niveau de connaissances thématiques dépend à la fois de celui de leur habileté linguistique mais aussi de la nature du processus qu'ils activent.

Par ailleurs, Kellogg $(1993,1994)$, qui classe dans les connaissances discursives le type de textes à produire et la nature du destinataire, a montré que les textes descriptifs et argumentatifs demandaient aux rédacteurs plus d'effort que les textes narratifs, et ce quel que soit le processus rédactionnel. Piolat, Roussey et Rous (1996) ont, de leur côté, mis en évidence que le degré de prestige associé au destinataire infléchissait l'effort cognitif ainsi que le pattern de mobilisation du processus de révision.

\subsubsection{Les stratégies interindividuelles}

Au lieu de chercher à qualifier a priori les habiletés rédactionnelles des participants à l'aide de procédés de classification (tests, épreuves d'habileté, etc.), Levy et Ransdell (1995) ont affronté 
directement la question de la variabilité fonctionnelle des rédacteurs. La nature et l'éventail de leurs observables leur ont permis de dégager des configurations fonctionnelles propres à chaque rédacteur. Ces configurations d'abord appelées «style » (1995) sont appelées « signatures » (1996) afin d'évoquer les signatures cursives personnalisées des individus, mettant ainsi encore plus en relief les variations interindividuelles. Elles ne rendent compte que de la façon dont les rédacteurs mobilisent les processus rédactionnels et non pas de l'effort cognitif qu'ils développent.

Levy et Ransdell $(1995,1996)$ constatent que ces signatures sont remarquablement stables tout au long des sessions d'écriture réalisées semaine après semaine. Ces observations permet tent de dépasser les classifications dichotomiques faites jusqu'alors pour rendre compte des différentes caractéristiques fonctionnelles des rédacteurs (cf. par ex. Galbraith, 1996 ; Scardamalia et Bereiter, 1991).

\subsection{EFFETS DES SITUATIONS DE RÉDACTION}

Au-delà de leurs différences interindividuelles, la méthode de la triple tâche permet aussi d'évaluer comment les rédacteurs peuvent s'adapter aux contraintes imposées par les conditions de production (étapes de composition rédactionnelles et médium de production). En effet, l'observation avec d'autres techniques que la triple tâche des rédacteurs a mis en évidence différentes étapes de composition et, pour chacune de ces étapes, différentes façon de procéder (cf. Piolat, 1999).

Kellogg $(1988,1990,1994)$ a testé expérimentalement l'impact de l'utilisation de divers types de stratégies de préécriture et d'organisation des informations (e.g. plan sous forme de sommaire écrit ou mental ; listes de thèmes, de réseaux conceptuels) et de stratégies de brouillonnage (brouillon élaboré ou ébauché). Pour l'essentiel, il a constaté que ce sont seulement les patterns de mobilisation des processus rédactionnels, et non pas l'effort cognitif, qui varient en fonction des types de stratégies de préécriture, d'organisation des informations et de brouillonnage. Les processus de planification et de révision autorisent et favorisent principalement ces ajustements stratégiques.

Par ailleurs, Kellogg et Mueller (1993) ont montré que l'utilisation du traitement de texte ne bouleverse pas le pattern 
de mobilisation des processus. Cependant, les rédacteurs utilisant un traitement de texte manifestent un effort cognitif beaucoup plus important que ceux qui écrivent à la main pour les processus de planification et de révision. Le degré d'expertise des utilisateurs du traitement de texte ne remet pas en cause ces observations. Olive, Piolat et Polge (1997) n'ont pas obtenu de différence d'effort cognitif en fonction du médium de production, qu'ils s'agissent de journalistes ou d'étudiants. En revanche, comparativement à ce qu'ils font en rédigeant à la main, les journalistes modifient leur pattern de mobilisation de processus (en révisant plus fréquemment) quand ils produisent (comme c'est leur cas habituellement) avec un traitement de texte.

\subsection{EFFORT COGNITIF ET QUALITÉ DE LA RÉDACTION}

Le texte constitue l'aboutissement de l'activité du rédacteur. Par hypothèse, un compromis devrait être mis en évidence entre l'effort cognitif, le pattern de mobilisation développé par le rédacteur et la qualité de son texte même s'il est particulièrement difficile d'évaluer la qualité des textes (Breetvelt, van den Bergh et Rijlaarsdam, 1996 ; Kellogg, 1987a, I994 ; Piolat et Pélissier, 1998 ; Schriver, 1989 ; Spencer et Fitzgerald, 1993). Kellogg (cf. sa synthèse de I994) a mis en évidence différents types de relations entre les caractéristiques des rédacteurs et/ou les contraintes de production et la qualité des textes produits. Il n'a pas apporté d'observation expérimentale sur les relations entre la qualité et l'effort. Olive et Piolat (1997) n'ont pas pu montrer de lien direct entre l'effort cognitif développé par des rédacteurs soumis au paradigme de Kellogg (1987b) et la qualité de leur texte (mesurée avec l'échelle SSQS de Levy et Ransdell, 1996).

Seuls Levy et Ransdell $($ I995, 1996) ont trouvé une relation positive entre les temps de réaction et la qualité des documents produits. Un effort plus grand est associé aux essais de meilleure qualité. De plus, ces auteurs ont montré que c'est le temps dévolu à réviser qui prédit le mieux la qualité, surtout lorsque la révision est faite dans la dernière étape de la rédaction. Ainsi, la révision prend moins du temps total de composition que d'autres sousprocessus mais elle détermine le succès de la rédaction. Enfin, leurs résultats suggèrent aussi que des documents de bonne qualité different non seulement en termes d'allocation mais, aussi, en 
termes de nombre et de distribution des périodes de sousprocessus. Levy et Ransdell $(1995,1996)$ mettent donc en évidence une relation entre la stratégie rédactionnelle particulière d'un individu (la signature) et la qualité du texte qu'il produit.

\subsection{BILAN}

Les différentes expériences qui viennent d'être évoquées mettent en jeu des facteurs propices à créer des situations de production qui exigent de la part des rédacteurs l'utilisation de types différents de connaissances (connaissances générales : thème, destinataire ; connaissances liées à la maîtrise de la langue écrite schémas de texte, habileté syntaxico-sémantique), ou encore des stratégies concernant la gestion de l'activité rédactionnelle dans son ensemble (faire un brouillon, se relire).

De façon synthétique, il est important de noter que ces sources potentielles de variation influencent diversement l'effort cognitif et/ou le pattern de mobilisation des processus. Trois groupes de facteurs peuvent être proposés

a) Ce sont les variations des connaissances générales du rédacteur qui font varier leur effort cognitif. L'ampleur des connaissances thématiques ou encore le degré de maîtrise des schémas de texte (narration vs argumentation vs description) constituent de bon candidat pour moduler l'effort cognitif que les rédacteurs devront développer pour composer un texte d'un genre donné sur un thème donné. Cependant, la mobilisation des processus ne paraît pas être influencée par ce type de facteur.

b) Ce sont, plutôt, les variations de stratégie du rédacteur qui font varier le pattern de mobilisation des processus rédactionnels. La façon dont le rédacteur organise son travail (faire tel ou tel type de brouillon ou de plan) transforme, durant une session de composition, sa façon de gérer ses processus rédactionnels, particulièrement la planification et la révision. En revanche, l'effort cognitif consenti ne paraît pas être influencé par ces facteurs.

c) Enfin, l'adaptation du rédacteur, en fonction de son degré d'habileté linguistique, à différentes contraintes de production (thème, destinataire) se manifestera par des ajustements à la fois de son effort cognitif et de ses séquences d'activation des processus rédactionnels. 


\section{CONCLUSION}

Le principal objectif de cet article était, après avoir décrit les variantes du paradigme de la triple tâche, d'en évaluer l'intérêt expérimental. Les résultats des recherches concernant une évaluation de l'impact (qualifié de « réactivité », d' « intrusion », etc.) des tâches ajoutées (réagir vite et qualifier ses pensées) sur la tâche principale de rédaction de texte ont été présentés. Force est de constater que le paradigme de la triple tâche est opérant. Il permet, sans bouleverser le décours de l'activité rédactionnelle, ni la qualité des textes produits, d'étudier les configurations selon lesquelles les rédacteurs mobilisent leurs processus rédactionnels ainsi que l'effort cognitif associé à cette mobilisation. Ce paradigme ne fournit des informations que sur les aspects délibérés des processus que sont la planification, la mise en texte et la révision, mais grâce à lui de nouvelles questions peuvent être posées. Ainsi, par exemple, un des problèmes qu'il faut élucider concerne les conditions d'installation des stratégies interindividuelles ainsi que leur marge d'adaptabilité aux différentes contraintes de production. Ces stratégies sont-elles le résultats de traitements plus ou moins efficaces ou sont-elles conditionnées par la capacité de la mémoire de travail des rédacteurs (Kellogg, 1999; Levy, 1997 ; McCutchen, 1996; Olive, 1999) ? L'utilisation, en rédaction de textes, du paradigme de la triple tâche sera, à ce sujet, certainement efficace et opportun.

\section{RÉSUMÉ}

Trois objectifs sont poursuivis dans cette note méthodologique concernant une des méthodes d'étude de la rédaction de textes. Le premier objectif est de rendre compte des variantes d'utilisation du paradigme de la triple tâche (rédi ger, réagir rapidement et verbaliser son activité mentale) mises au point par Kellogg (1987b) ainsi que Levy et Ransdell (1994, 1995). Cette méthode permet de statuer sur l'effort cognitif associé à chacun des processus rédactionnels (planifier, mettre en texte et réviser) en recueillant les temps de réaction associés à chacun de ces processus. Elle permet aussi d'étudier l'évolution de la mobilisation des processus rédactionnels tout au long de la tâche de production verbale écrite. Le deuxième objectif consiste à éprouver la validité de ce paradigme. À partir de recherches qui ont testé l'impact de la tâche de réaction rapide et de la verbalisation sur la tâche rédactionnelle, il est montré que 
l'adjonction de ce paradigme ne dénature pas la tâche de rédaction de texte. Le troisième objectif est de faire un rapide bilan des données expérimentales ainsi obtenues concernant les caractéristiques fonctionnelles de la rédaction, en soulignant les pistes de recherches ainsi ouvertes.

Mots clés : rédaction, processus, effort cognitif, mobilisation, paradigme de la triple tâche.

\section{BIBLIOGRAPHIE}

Baddeley A. D., Hitch G. J. - (1974) Working memory, in G. Bower (Edit.), The psychology of learning and motivation, New York, Academic Press, vol. 8, 47-90.

Barbier M. L., Piolat A., Roussey J.-Y. - (1998) Effet du traitement de texte et des correcteurs sur la maîtrise de l'orthographe et de la grammaire en langue seconde, Revue Française de Pédagogie, 122, 83-98.

Bock J. K. - (1996) Language production : Methods and methodologies, Psychonomic Bulletin and Reviews, 3 (4), 395-421.

Bonin P., Fayol M. - (1996) L'étude en temps réel de la production du langage écrit : pourquoi et comment?, Études de Linguistique Appliquée, 101, 8-19.

Breetvelt L, van den Bergh H., Rijlaarsdam G. - (1994) Relations between writing processes and text quality : When and how, Cognition and Instruction, 12, 103-123.

Breetvelt L, van den Bergh H., Rijlaarsdam G. - (1996) Rereading and generating and their relation to text quality. An application of multilevel analysis on writing process data, in G. Rijlaarsdam, H. van den Bergh et M. Couzijn (Edit.), Theories, models and methodology in writing research, Amsterdam, Amsterdam University Press, 10-20.

Burtis P. .J., Bereiter C., Scardamalia M., Tetroe J. - (1983) The development of planning in writing, in G. Wells et B. M. Kroll (Edit.), Explorations in the development of writing, New York, Wiley \& Sons, 153-174.

Camus J.-F. - (1996). La psychologie cognitive de l'attention, Paris, Armand Colin.

Coirier P., Gaonac'h D., Passerault J.-M. - (1996) Psycholinguistique textuelle approche cognitive de la compréhension et de la production des textes, Paris, Armand Colin.

Cooper M. R. - (1983) Procedures for describing written texts, in P. Mosenthal, L. Tamor et S. A. Walmsley (Edit.), Research on writing. Principles and methods, New York (N,1), Longman, 287-313.

Cooper M., Holzman M. - (1983) Talking about protocols, College Composition and Communication, 34, 284-293.

Cooper M., Holzman M. - (1985) Reply by Marilyn Cooper and Michael Holzman, College Composition and Communication, 36, 97-100.

Cumming A. - (1994) Writing expertise and second language proficiency, in A. Cumming (Edit.), Bilingual performance in reading and writing, Amsterdam, John Benjamins, 173-221.

Ericsson K. A., Simon H. A. - (1993) Protocol analysis : Verbal reports as data, Cambridge, MIT Press.

Fayol M. - (1997) Des idées au texte : psychologie cognitive de la production verbale, orale et écrite, Paris, PUF. 
Fisk A. D., Derrick W. L., Schneider W. - (1986-1987) A methodological assessment and evaluation of dual-task paradigms, Current Psychological Research and Reviews, 5, 315-327.

Flower L., Hayes J. R. - (1980) The dynamics of composing : Making plans and juggling with constraints, in L. Gregg et E. Steinberg (Edit.), Cognitive process in writing, Hillsdale (N.1), Lawrence Erlbaum, 31-50.

Flower L., Hayes J. R. - (1981) The pregnant pause : An inquiry into the nature of planning, Research in the Teaching of English, 15 (3), 229-243.

Ford M., Holmes V. - (1978) Planning units and syntax in sentence production, Cognition, 6, 35-53.

Foulin J. N. - (1995) Pauses et débits : les indicateurs temporels de la production écrite, L'Année Psychologique, 95, 483-504.

Galbraith D. - (1996) Self-monitoring, discovery through writing and individual differences in drafting strategy, in G. Rijlaarsdam, H. van den Bergh et M. Couzjin (Edit.), Theories, models and methodology in writing research, Amsterdam, Amsterdam University Press, 121-141.

Gathercole S. E., Baddeley A. D. - (1993) Working memory and language, Hove, Lawrence Erlbaum.

Gernsbacher M. A. - (1994) Handbook of psycholinguistics, New York, Academic Press.

Gufoni V. - (1996) Les protocoles verbaux comme méthode d'étude de la production écrite : approche critique, Etudes de Linguistique Appliquée, 101, 20-32.

Hayes J. R., Flower L. S. - (1980) Identifying the organization of writing process, in L. W. Gregg et E. R. Steinberg (Edit.), Cognitive processes in writing, Hillsdale (NJ), Lawrence Erlbaum, 3-30.

Hayes J. R., Flower L. S. - (1983) Uncovering cognitive process in writing An introduction to protocol analysis, in P. Rosenthal, L. Tamor et S. A. Walmsley (Edit.), Research on writing : Principles and methods, New York, Longman, 207-221.

Hayes J. R., Flower 1.. S., Schriver K., Stratman J., Carey L. - (1987) Cognitive processes in revision, in S. Rosenberg (Edit.), Reading, writing and language learning : Advances in applied psycholinguistics, Cambridge (MA), Cambridge University Press, vol. 11, 176-240.

Hupet M., Schelstraete M. A., Demaeght N., Fayol M. - (1996) Les erreurs d'accord sujet-verbe en production écrite, L'Année Psychologique, 96, 587610.

Janssen D., van Waes L., van den Bergh H. - (1996) Effects of thinking aloud on writing processes, in C. M. Levy et S. E. Ransdell (Edit.), The science of writing : Theories, methods, individual differences and applications, Mahwah (NJ), Lawrence Erlbaum, 233-250.

Just M. A., Carpenter P. A. - (1992) A capacity theory of comprehension Individual differences in working memory, Psychological Review, 99, 122149.

Kahneman D. - (1973) Attention and effort, Englewood Cliffs (NJ), PrenticeHall.

Kaufer D. S., Hayes J. R., Flower L. S. - (1986) Composing written sentences, Research in the Teaching of English, 20, 121-140.

Kellogg R. T. - (1987a) Writing performance : Effect of cognitive strategies, Written Communication, 4, 269-298.

Kellogg R. T. - (19876) Effects of topic knowledge on the allocation of processing time and cognitive effort to writing processes, Memory and Cognition, 15 (3), 256-266. 
Kellogg R. T.-(1988) Attentional overload and writing performance : Effects of rough draft and outline strategies, Journal of Experimental Psychology Learning, Memory and Cognition, 14 (2), 355-365.

Kellogg R. T. - (1990) Effectiveness of prewriting strategies as a function of task demands, American Journal of Psychology, 103 (3), 327-342.

Kellogg R. T. - (1993) Observations on the psychology of thinking and writing, Composition Studies, 21, 3-41.

Kellogg R. T. - (1994) The psychology oj'writing, New York, Oxford University Press.

Kellogg R. T. - (1996) A model of working memory in writing, in C. M. Levy et S. E. Ransdell (Edit.), The science of writing: Theories, methods, individual differences and applications, Mahwah (NJ), Laurence Erlbaum, 57-71.

Kellogg R. T. - (1999) Components of working memory in text production, in M. Torrance et G. Jeffery (Edit.), The cognitive demands of writing : Processing capacity and working memory effects in text production. Amsterdam, Amsterdam University Press, 43-62.

Kellogg R. T., Mueller S. - (1993) Performance amplification and process restructuring in computer-based writing, International Journal of ManMachine Studies, 39, 33-49.

Kempen G., Hoenkamp E. - (1987) A incremental procedural grammar for sentence formulation, Cognitive Science, 11, 201-258.

Kerr B. - (1973) Processing demands during mental operations, Memory and Cognition, 1, 401-412.

Levelt W. J. M. - (1989) Speaking : From intention to articulation, Cambridge (MA), MIT Press.

Levy C. M. - (1997) The « $\mathrm{R} »$ that psychology forgot : Research on writing processes, Behavior Research Methods, Instruments, and Computers, 29, 137-145.

Levy C. M., Ransdell S. E. - (1994) Computer-aided protocol analysis of writing processes, Behavior Research Methods, Instruments, and Computers, 26, 219-223.

Levy C. M., Ransdell S. E. - (1995) Is writing as difficult as it seems ?, Memory and Cognition, 23, 6, 767-779.

Levy C. M., Ransdell S. E. - (1996) Writing signatures, in C. M. Levy et S. Ransdell (Edit.), The science oj'writing. Theories, methods, individual differences and applications, Mahwah (NJ), Lawrence Erlbaum, 149-161.

Levy C. M., Marek J. P., Lea J. - (1996) Concurrent and retrospective protocols in writing research, in G. Rijlaarsdam, H. van den Bergh et M. Couzjin (Edit.), Writing research : Theories, models and methodology, Amsterdam, Amsterdam University Press, 542-556.

McCutchen D. - (1996) A capacity theory of writing : Working memory in composition, Educational Psychology Review, 8 (3), 299-325.

Navon D. (1984) Resources - A theoretical soup stone ?, Psychological Review, 91 (2), 216-234.

Navon D., Gopher D. - (1979) On the economy of human processing system, Psychological Review, 86, 214-255.

Olive T. - (1997) Validité de la méthode de TR et de rétrospection dirigée pour étudier la production de texte, Informations in Cognito, 9, 19-30.

Olive T. - (1999) Exploring the activation of the writing components with a composition and a copy task, in M.-F. Crété et E. Espéret (Edit.), Proceedings of the 1998 European Writing Conference, Poitiers, Université de Poitiers, 65-70.

Olive T., Piolat A. - (1997) Existe-t-il un lien entre l'effort cognitif développé par 
des rédacteurs et la qualité du texte ?, Communication au Congrès annuel de la Société Française de Psychologie (mai), Nice.

Olive T., Piolat A., Polge M. - (1997) An evaluation of mental effort of professional and experienced writers during the use of a word processor, Communication â 7th European Conference for Research on Learning and Instruction (août), Athènes.

Olive T., Piolat A., Roussey J.-Y. - (1997a) Effort cognitif et mobilisation des processus : effet de l'habileté rédactionnelle et du niveau de connaissances, in D. Mellier et A. Voin Hofe (Edit.), Attention et contrôle cognitif : Mécanismes, développement des habiletés, pathologies, Rouen, Publications de l'Université de Rouen, 71-58.

Olive T., Piolat A., Roussey J. Y. - (1997b) The effect of expertise and knowledge on cognitive effort associated to writing processes, in J. L. Rodriguez Illera et L. Tolchinsky (Edit.), Proceedings of the 1996 European Writing Conferences, Barcelona, ICE - Universitat de Barcelona, 1-29.

Pashler H. - (1984) Processing stages in overlapping tasks : Evidence for a central bottleneck, Journal of Experimental Psychology : Human Perception and Performance, 10, 358-377.

Pélissier A., Piolat A. - (1998) Are writing processes activated sequentially or in parallel ? The appropriate way to use Kellogg's three task method, in M.-F. Crété et E. Espéret (Edit.), Proceedings of the 1998 European Writing Conference, Poitiers, Université de Poitiers, 71-76.

Penningroth S. L., Rosenberg S. - (1995) Effects of a high information processing load on the writing process and the story written, Applied Psycholinguistics, 16, 189-210.

Piolat A. - (1999) Planning and text quality among undergraduate students Findings and questions, in D. Galbraith et M. Torrance (Edit.), Knowing what to write : Conceptual processes in text production, Amsterdam, Amsterdam University Press.

Piolat A., Blaye A. - (1991) Effects of word processing and writing aids on revision processes, in M. Carretero, M. Pope, R. Simons et J. I. Pozo (Edit.), Learning and instruction : European research in an international context, Oxford, Pergamon Press, vol. III, 379-399.

Piolat A., Pélissier A. - (1998) Approche cognitive de la rédaction de textes contraintes théoriques et méthodes de recherches, in A. Piolat et A. Pélissier (Édit.), La rédaction de texte : approche cognitive, Neuchâtel, Delachaux \& Niestlé, 225-269.

Piolat A., Isnard N., Della Valle V. - (1993) Traitement de texte et stratégies rédactionnelles, Le Travail Humain, 56 (3), 79-99.

Piolat A., Roussey J.-Y., Rous P. - (1996) Effects of attention directed to different texts on the mental effort related to writing processes, Communication â 1996 European Writing Conference (octobre), Barcelone.

Piolat A., Roussey J.-Y., Olive T., Farioli F. - (1996) Charge mentale et mobilisation des processus rédactionnels : examen de la procédure de Kellogg, Psychologie Française, 41, 339-354.

Piolat A., Olive T., Roussey J.-Y., Thunin 0., Ziegler J. C. - (1999) Scriptkell : A tool for measuring cognitive effort and time processing in writing and other complex cognitive activities, Behavior Research Methods, Instruments, and Computers, 31 (1), 113-121.

Posner M. I. - (1978) Chronometric explorations of' mind, Hillsdale (NJ), Lawrence Erlbaum.

Power M. J. - (1985) Sentence production and working memory, The Quarterly Journal of Experimental Psychology, 37A, 367-385. 